\title{
Coefficient Bounds for Subclasses of Biunivalent Functions Associated with the Chebyshev Polynomials
}

\author{
Hatun Özlem Güney, ${ }^{1}$ G. Murugusundaramoorthy, ${ }^{2}$ and K. Vijaya ${ }^{2}$ \\ ${ }^{1}$ Faculty of Science, Department of Mathematics, Dicle University, 21280 Diyarbakir, Turkey \\ ${ }^{2}$ School of Advanced Sciences, VIT University, Vellore 632014, India \\ Correspondence should be addressed to Hatun Özlem Güney; ozlemg@dicle.edu.tr
}

Received 28 August 2017; Accepted 26 October 2017; Published 26 November 2017

Academic Editor: Yan Xu

Copyright ( 2017 Hatun Özlem Güney et al. This is an open access article distributed under the Creative Commons Attribution License, which permits unrestricted use, distribution, and reproduction in any medium, provided the original work is properly cited.

We introduce and investigate new subclasses of biunivalent functions defined in the open unit disk, involving Sălăgean operator associated with Chebyshev polynomials. Furthermore, we find estimates of the first two coefficients of functions in these classes, making use of the Chebyshev polynomials. Also, we give Fekete-Szegö inequalities for these function classes. Several consequences of the results are also pointed out.

\section{Introduction}

Let $\mathscr{A}$ denote the class of analytic functions of the form

$$
f(z)=z+\sum_{n=2}^{\infty} a_{n} z^{n}
$$

normalized by the conditions $f(0)=0=f^{\prime}(0)-1$ defined in the open unit disk

$$
\triangle=\{z \in \mathbb{C}:|z|<1\} .
$$

Let $\mathcal{S}$ be the subclass of $\mathscr{A}$ consisting of functions of form (1) which are also univalent in $\triangle$. Let $\mathcal{S}^{*}(\alpha)$ and $\mathscr{K}(\alpha)$ denote the well-known subclasses of $\mathcal{S}$, consisting of starlike and convex functions of order $\alpha(0 \leq \alpha<1)$, respectively.

The Koebe one-quarter theorem [1] ensures that the image of $\triangle$ under every univalent function $f \in \mathscr{A}$ contains a disk of radius $1 / 4$. Thus every univalent function $f$ has an inverse $f^{-1}$ satisfying

$$
\begin{aligned}
& f^{-1}(f(z))=z, \quad(z \in \Delta) \\
& f\left(f^{-1}(w)\right)=w \quad\left(|w|<r_{0}(f), r_{0}(f) \geq \frac{1}{4}\right) .
\end{aligned}
$$

A function $f \in \mathscr{A}$ is said to be biunivalent in $\triangle$ if both $f$ and $f^{-1}$ are univalent in $\triangle$. Let $\Sigma$ denote the class of biunivalent functions defined in the unit disk $\triangle$. Since $f \in \Sigma$ has the Maclaurin series given by (1), a computation shows that its inverse $g=f^{-1}$ has the expansion

$$
g(w)=f^{-1}(w)=w-a_{2} w^{2}+\left(2 a_{2}^{2}-a_{3}\right) w^{3}+\cdots .
$$

An analytic function $f$ is subordinate to an analytic function $g$, written as $f(z) \prec g(z)$, provided there is an analytic function $w$ defined on $\triangle$ with $w(0)=0$ and $|w(z)|<$ 1 satisfying $f(z)=g(w(z))$.

Chebyshev polynomials, which are used by us in this paper, play a considerable role in numerical analysis. We know that the Chebyshev polynomials are four kinds. The most of books and research articles related to specific orthogonal polynomials of Chebyshev family contain essentially results of Chebyshev polynomials of first and second kinds $T_{n}(x)$ and $U_{n}(x)$ and their numerous uses in different applications; see Doha [2] and Mason [3].

The well-known kinds of the Chebyshev polynomials are the first and second kinds. In the case of real variable $x$ on $(-1,1)$, the first and second kinds are defined by

$$
\begin{aligned}
& T_{n}(x)=\cos n \theta, \\
& U_{n}(x)=\frac{\sin (n+1) \theta}{\sin \theta},
\end{aligned}
$$


where the subscript $n$ denotes the polynomial degree and $x=$ $\cos \theta$. We consider the function

$$
\Phi(z, t)=\frac{1}{1-2 t z+z^{2}} .
$$

We note that if $t=\cos \alpha, \alpha \in(-\pi / 3, \pi / 3)$, then for all $z \in \Delta$

$$
\begin{aligned}
\Phi(z, t) & =\frac{1}{1-2 t z+z^{2}}=1+\sum_{n=1}^{\infty} \frac{\sin (n+1) \alpha}{\sin \alpha} z^{n} \\
& =1+2 \cos \alpha z+\left(3 \cos ^{2} \alpha-\sin ^{2} \alpha\right) z^{2}+\cdots .
\end{aligned}
$$

Thus, we write

$$
\Phi(z, t)=1+U_{1}(t) z+U_{2}(t) z^{2}+\cdots
$$

$$
(z \in \Delta, t \in(-1,1)),
$$

where $U_{n-1}=\sin (n \arccos t) / \sqrt{1-t^{2}}$, for $n \in \mathbb{N}$, are the second kind of the Chebyshev polynomials. Also, it is known that

$$
\begin{aligned}
& U_{n}(t)=2 t U_{n-1}(t)-U_{n-2}(t), \\
& U_{1}(t)=2 t ; \\
& U_{2}(t)=4 t^{2}-1, \\
& U_{3}(t)=8 t^{3}-4 t, \ldots .
\end{aligned}
$$

The Chebyshev polynomials $T_{n}(t), t \in[-1,1]$, of the first kind have the generating function of the form

$$
\sum_{n=0}^{\infty} T_{n}(t) z^{n}=\frac{1-t z}{1-2 t z+z^{2}} \quad(z \in \Delta) .
$$

All the same, the Chebyshev polynomials of the first kind $T_{n}(t)$ and the second kind $U_{n}(t)$ are well connected by the following relationship:

$$
\begin{aligned}
\frac{d T_{n}(t)}{d t} & =n U_{n-1}(t), \\
T_{n}(t) & =U_{n}(t)-t U_{n-1}(t), \\
2 T_{n}(t) & =U_{n}(t)-U_{n-2}(t) .
\end{aligned}
$$

Several authors have introduced and investigated subclasses of biunivalent functions and obtained bounds for the initial coefficients (see [4-10]). In [11], making use of the Sălăgean [12] differential operator,

$$
D^{k}: \mathscr{A} \longrightarrow \mathscr{A}
$$

defined by

$$
\begin{aligned}
& \mathscr{D}^{0} f(z)=f(z), \\
& \mathscr{D}^{1} f(z)=\mathscr{D} f(z)=z f^{\prime}(z), \\
& \mathscr{D}^{k} f(z)=\mathscr{D}\left(\mathscr{D}^{k-1} f(z)\right)=z\left(\mathscr{D}^{k-1} f(z)\right)^{\prime}, \\
& \qquad k \in \mathbb{N}=\{1,2,3, \ldots\}, \\
& \mathscr{D}^{k} f(z)=z+\sum_{n=2}^{\infty} n^{k} a_{n} z^{n}, \quad k \in \mathbb{N}_{0}=\mathbb{N} \cup\{0\},
\end{aligned}
$$

and further for functions $g$ of the form (4) Vijaya et al. [11] (also see [13]) defined

$$
D^{k} g(w)=w-a_{2} 2^{k} w^{2}+\left(2 a_{2}^{2}-a_{3}\right) 3^{k} w^{3}+\cdots
$$

and introduced two new subclasses of biunivalent functions. In this paper, we use Chebyshev polynomials to obtain the estimates on the coefficients $\left|a_{2}\right|$ and $\left|a_{3}\right|$.

\section{Biunivalent Function Classes $\mathscr{M}_{\Sigma}^{k}(\lambda, \Phi(z, t))$ and $\mathscr{F}_{\Sigma}^{k}(\beta, \Phi(z, t))$}

Motivated by recent works of Altinkaya and Yalcin [14] (also see [15]) and recent studies on biunivalent functions involving Sălăgean operator [11, 13], in this section, we introduce two new subclasses of $\Sigma$ associated with Chebyshev polynomials and obtain the initial Taylor coefficients $\left|a_{2}\right|$ and $\left|a_{3}\right|$ for the function classes by subordination.

Definition 1. For $0 \leq \lambda \leq 1$ and $t \in(-1,1)$ a function $f \in$ $\Sigma$ of form (1) is said to be in the class $\mathscr{M}_{\Sigma}^{k}(\lambda, \Phi(z, t))$ if the following subordination holds:

$$
\begin{aligned}
& (1-\lambda) \frac{D^{k+1} f(z)}{D^{k} f(z)}+\lambda \frac{D^{k+2} f(z)}{D^{k+1} f(z)} \prec \Phi(z, t), \\
& (1-\lambda) \frac{D^{k+1} g(w)}{D^{k} g(w)}+\lambda \frac{D^{k+2} g(w)}{D^{k+1} g(w)} \prec \Phi(w, t),
\end{aligned}
$$

where $z, w \in \Delta$ and $g$ is given by (4).

We note that by specializing the parameters $\lambda$ and suitably fixing the values for $k$ in Definition 1, we introduce (had not been studied so far) the following new subclasses of $\Sigma$ as listed below.

Remark 2. Supposing $f(z) \in \Sigma$ and $t \in(-1,1)$, then we denote
(1) $\mathscr{M}_{\Sigma}^{k}(0, \Phi(z, t)) \equiv \delta_{\Sigma}^{k}(\Phi(z, t))$,
(2) $\mathscr{M}_{\Sigma}^{k}(1, \Phi(z, t)) \equiv \mathscr{K}_{\Sigma}^{k}(\Phi(z, t))$,
(3) $\mathscr{M}_{\Sigma}^{0}(0, \Phi(z, t))=\mathcal{S}_{\Sigma}^{*}(\Phi(z, t))$,
(4) $\mathscr{M}_{\Sigma}^{0}(1, \Phi(z, t))=\mathscr{K}_{\Sigma}(\Phi(z, t))$.

Due to Frasin and Aouf [16] and Panigarhi and Murugusundaramoorthy [17] (also see [11, 13]) we define the following new subclass involving the Sălăgean operator [12].

Definition 3. For $0 \leq \beta \leq 1$ and $t \in(-1,1)$ a function $f \in \Sigma$ of form (1) is said to be in the class $\mathscr{F}_{\Sigma}^{k}(\beta, \Phi(z, t))$ if the following subordination holds:

$$
\begin{aligned}
& (1-\beta) \frac{D^{k} f(z)}{z}+\beta\left(D^{k} f(z)\right)^{\prime}<\Phi(z, t), \\
& (1-\beta) \frac{D^{k} g(w)}{w}+\beta\left(D^{k} g(w)\right)^{\prime}<\Phi(w, t),
\end{aligned}
$$

where $z, w \in \Delta, g=f^{-1}, D^{k} f(z)$ and $D^{k} g(w)$ are given by (4), (15), and (16), respectively. 
In Definition 3, by specializing the parameters $\beta$ and suitably fixing the values for $k$ (had not been studied so far) the following new subclasses of $\Sigma$ are as listed below.

Remark 4. Supposing $f(z) \in \Sigma$ and $t \in(-1,1)$, then we denote

(1) $\mathscr{F}_{\Sigma}^{k}(0, \Phi(z, t)) \equiv \mathscr{R}_{\Sigma}^{k}(\Phi(z, t))$,

(2) $\mathscr{F}_{\Sigma}^{k}(1, \Phi(z, t)) \equiv \mathscr{H}_{\Sigma}^{k}(\Phi(z, t))$,

(3) $\mathscr{F}_{\Sigma}^{0}(\beta, \Phi(z, t)) \equiv \mathscr{F}_{\Sigma}(\beta, \Phi(z, t))$,

(4) $\mathscr{F}_{\Sigma}^{0}(1, \Phi(z, t)) \equiv \mathscr{H}_{\Sigma}^{0}(\Phi(z, t))$.

In the following theorems we determine the initial Taylor coefficients $\left|a_{2}\right|$ and $\left|a_{3}\right|$ for the function classes $f \in$ $\mathscr{M}_{\Sigma}^{k}(\lambda, \Phi(z, t))$ and $f \in \mathscr{F}_{\Sigma}^{k}(\beta, \Phi(z, t))$.

Theorem 5. Let $f$ given by (1) be in the class $\mathscr{M}_{\Sigma}^{k}(\lambda, \Phi(z, t))$ and $t \in(0,1)$. Then

$$
\begin{aligned}
& \left|a_{2}\right| \\
& \leq \frac{2 t \sqrt{2 t}}{\sqrt{\left|\left[2(1+2 \lambda) 3^{k}-\left(\lambda^{2}+5 \lambda+2\right) 2^{2 k}\right] 4 t^{2}+(1+\lambda)^{2} 2^{2 k}\right|}}, \\
& \left|a_{3}\right| \leq \frac{4 t^{2}}{(1+\lambda)^{2} 2^{2 k}+\frac{t}{(1+2 \lambda) 3^{k}},}
\end{aligned}
$$

where $0 \leq \lambda \leq 1$ and $t \neq 1 / \sqrt{2}$.

Proof. Let $f \in \mathscr{M}_{\Sigma}^{k}(\lambda, \Phi(z, t))$ and $g=f^{-1}$. Considering (17), we have

$$
\begin{aligned}
& (1-\lambda) \frac{D^{k+1} f(z)}{D^{k} f(z)}+\lambda \frac{D^{k+2} f(z)}{D^{k+1} f(z)}=\Phi(z, t), \\
& (1-\lambda) \frac{D^{k+1} g(w)}{D^{k} g(w)}+\lambda \frac{D^{k+2} g(w)}{D^{k+1} g(w)}=\Phi(w, t) .
\end{aligned}
$$

Define the functions $u(z)$ and $v(w)$ by

$$
\begin{aligned}
& u(z)=c_{1} z+c_{2} z^{2}+\cdots, \\
& v(w)=d_{1} w+d_{2} w^{2}+\cdots
\end{aligned}
$$

which are analytic in $\Delta$ with $u(0)=0=v(0)$ and $|u(z)|<1$, $|v(w)|<1$, for all $z \in \Delta$. It is well known that

$$
\begin{aligned}
& |u(z)|=\left|c_{1} z+c_{2} z^{2}+\cdots\right|<1, \\
& |v(w)|=\left|d_{1} w+d_{2} w^{2}+\cdots\right|<1,
\end{aligned}
$$

$$
z, w \in \triangle
$$

and then

$$
\begin{aligned}
& \left|c_{j}\right| \leq 1, \\
& \left|d_{j}\right| \leq 1 \\
& \quad \forall j \in \mathbb{N} .
\end{aligned}
$$

Using (22) and (23) in (20) and (21), respectively, we have

$$
\begin{aligned}
(1 & -\lambda) \frac{D^{k+1} f(z)}{D^{k} f(z)}+\lambda \frac{D^{k+2} f(z)}{D^{k+1} f(z)} \\
= & 1+U_{1}(t) u(z)+U_{2}(t) u^{2}(z)+\cdots, \\
(1 & -\lambda) \frac{D^{k+1} g(w)}{D^{k} g(w)}+\lambda \frac{D^{k+2} g(w)}{D^{k+1} g(w)} \\
= & 1+U_{1}(t) v(w)+U_{2}(t) v^{2}(w)+\cdots .
\end{aligned}
$$

In light of (1), (4), (10), (15), and (16) and from (26), we have

$$
\begin{aligned}
1+ & (1+\lambda) 2^{k} a_{2} z+\left[2(1+2 \lambda) 3^{k} a_{3}-(1+3 \lambda) 2^{2 k} a_{2}^{2}\right] \\
\cdot & z^{2}+\cdots=1+U_{1}(t) c_{1} z+\left[U_{1}(t) c_{2}+U_{2}(t) c_{1}^{2}\right] \\
& \cdot z^{2}+\cdots, \\
1- & (1+\lambda) 2^{k} a_{2} w+\left\{\left[(8 \lambda+4) 3^{k}-(3 \lambda+1) 2^{2 k}\right] a_{2}^{2}\right. \\
& \left.-2(1+2 \lambda) 3^{k} a_{3}\right\} w^{2}+\cdots=1+U_{1}(t) d_{1} w \\
& +\left[U_{1}(t) d_{2}+U_{2}(t) d_{1}^{2}\right] w^{2}+\cdots .
\end{aligned}
$$

This yields the following relations:

$$
\begin{aligned}
& (1+\lambda) 2^{k} a_{2}=U_{1}(t) c_{1}, \\
& -(1+3 \lambda) 2^{2 k} a_{2}^{2}+2(1+2 \lambda) 3^{k} a_{3} \\
& \quad=U_{1}(t) c_{2}+U_{2}(t) c_{1}^{2}, \\
& -(1+\lambda) 2^{k} a_{2}=U_{1}(t) d_{1}, \\
& \left(4(1+2 \lambda) 3^{k}-(1+3 \lambda) 2^{2 k}\right) a_{2}^{2}-2(1+2 \lambda) 3^{k} a_{3} \\
& \quad=U_{1}(t) d_{2}+U_{2}(t) d_{1}^{2} .
\end{aligned}
$$

From (28) and (30) it follows that

$$
\begin{aligned}
c_{1} & =-d_{1}, \\
2(1+\lambda)^{2} 2^{2 k} a_{2}^{2} & =U_{1}^{2}(t)\left(c_{1}^{2}+d_{1}^{2}\right) .
\end{aligned}
$$

Adding (29) to (31) and using (33), we obtain

$$
\begin{aligned}
& a_{2}^{2} \\
& =\frac{U_{1}^{3}(t)\left(c_{2}+d_{2}\right)}{2\left[\left\{2(1+2 \lambda) 3^{k}-(1+3 \lambda) 2^{2 k}\right\} U_{1}^{2}(t)-(1+\lambda)^{2} 2^{2 k} U_{2}(t)\right]} .
\end{aligned}
$$

Applying (25) to the coefficients $c_{2}$ and $d_{2}$ and using (10) we have

$$
\begin{aligned}
& \left|a_{2}\right| \\
& \leq \frac{2 t \sqrt{2 t}}{\sqrt{\left|\left[2(1+2 \lambda) 3^{k}-\left(\lambda^{2}+5 \lambda+2\right) 2^{2 k}\right] 4 t^{2}+(1+\lambda)^{2} 2^{2 k}\right|}} .
\end{aligned}
$$


By subtracting (31) from (29) and using (32) and (33), we get

$$
a_{3}=\frac{U_{1}^{2}(t)\left(c_{1}^{2}+d_{1}^{2}\right)}{2(1+\lambda)^{2} 2^{2 k}}+\frac{U_{1}\left(c_{2}-d_{2}\right)}{4(1+2 \lambda) 3^{k}} .
$$

Using (10), once again applying (25) to the coefficients $c_{1}, c_{2}$, $d_{1}$, and $d_{2}$, we get

$$
\left|a_{3}\right| \leq \frac{4 t^{2}}{(1+\lambda)^{2} 2^{2 k}}+\frac{t}{(1+2 \lambda) 3^{k}} .
$$

By taking $\lambda=0$ or $\lambda=1$ and $t \in(0,1)$, one can easily state the estimates $\left|a_{2}\right|$ and $\left|a_{3}\right|$ for the function classes $\mathscr{M}_{\Sigma}^{k}(0, \Phi(z, t))=\delta_{\Sigma}^{k}(\Phi(z, t))$ and $\mathscr{M}_{\Sigma}^{k}(1, \Phi(z, t))=$ $\mathscr{K}_{\Sigma}^{k}(\Phi(z, t))$, respectively.

Remark 6. Let $f$ given by (1) be in the class $\delta_{\Sigma}^{k}(\Phi(z, t))$. Then

$$
\begin{aligned}
& \left|a_{2}\right| \leq \frac{2 t \sqrt{2 t}}{\sqrt{\left|\left[3^{k}-2^{2 k}\right] 8 t^{2}+2^{2 k}\right|}}, \\
& \left|a_{3}\right| \leq \frac{4 t^{2}}{2^{2 k}}+\frac{t}{3^{k}} .
\end{aligned}
$$

Remark 7. Let $f$ given by (1) be in the class $\mathscr{K}_{\Sigma}^{k}(\Phi(z, t))$. Then

$$
\begin{aligned}
& \left|a_{2}\right| \leq \frac{2 t \sqrt{2 t}}{\sqrt{\left|\left[3^{k+1}-2^{2(k+1)}\right] 8 t^{2}+2^{2(k+1)}\right|}}, \\
& \left|a_{3}\right| \leq \frac{t^{2}}{2^{2 k}}+\frac{t}{3^{k+1}} .
\end{aligned}
$$

For $k=0$, Theorem 5 yields the following corollary.

Corollary 8. Let $f$ given by (1) be in the class $\mathscr{M}_{\Sigma}^{0}(\lambda, \Phi(z, t))$. Then

$$
\begin{aligned}
& \left|a_{2}\right| \leq \frac{2 t \sqrt{2 t}}{\sqrt{\left|(1+\lambda)^{2}-\left(\lambda^{2}+\lambda\right) 4 t^{2}\right|}}, \\
& \left|a_{3}\right| \leq \frac{4 t^{2}}{(1+\lambda)^{2}}+\frac{t}{(1+2 \lambda)},
\end{aligned}
$$

where $0 \leq \lambda \leq 1$ and $t \neq 1 / \sqrt{2}$.

By taking $k=0$ in the above remarks we get the estimates $\left|a_{2}\right|$ and $\left|a_{3}\right|$ for the function classes $\mathcal{S}_{\Sigma}^{*}(\Phi(z, t))$ and $\mathscr{K}_{\Sigma}(\Phi(z, t))$.

Remark 9. Let $f$ given by (1) be in the class $\delta_{\Sigma}^{k}(\Phi(z, t))$. Then

$$
\begin{aligned}
& \left|a_{2}\right| \leq 2 t \sqrt{2 t}, \\
& \left|a_{3}\right| \leq 4 t^{2}+t .
\end{aligned}
$$

Remark 10. Let $f$ given by (1) be in the class $\mathscr{K}_{\Sigma}^{k}(\Phi(z, t))$. Then, for $t \neq 1 / \sqrt{2}$,

$$
\begin{aligned}
& \left|a_{2}\right| \leq \frac{2 t \sqrt{2 t}}{\sqrt{\left|4-8 t^{2}\right|}}, \\
& \left|a_{3}\right| \leq t^{2}+\frac{t}{3} .
\end{aligned}
$$

Theorem 11. Let $f$ given by (1) be in the class $\mathscr{F}_{\Sigma}^{k}(\beta, \Phi(z, t))$ and $t \in(0,1)$. Then

$$
\begin{aligned}
& \left|a_{2}\right| \\
& \leq \frac{2 t \sqrt{2 t}}{\sqrt{\left|\left[(1+2 \beta) 3^{k}-(1+\beta)^{2} 2^{2 k}\right] 4 t^{2}+(1+\beta)^{2} 2^{2 k}\right|}}, \\
& \left|a_{3}\right| \leq \frac{4 t^{2}}{(1+\beta)^{2} 2^{2 k}}+\frac{2 t}{(1+2 \beta) 3^{k}} .
\end{aligned}
$$

Proof. Proceeding as in the proof of Theorem 5 we can arrive at the following relations:

$$
\begin{aligned}
& (1+\beta) 2^{k} a_{2}=U_{1}(t) c_{1}, \\
& (1+2 \beta) 3^{k} a_{3}=U_{1}(t) c_{2}+U_{2}(t) c_{1}^{2}, \\
& -(1+\beta) 2^{k} a_{2}=U_{1}(t) d_{1}, \\
& 2(1+2 \beta) 3^{k} a_{2}^{2}-(1+2 \beta) 3^{k} a_{3} \\
& \quad=U_{1}(t) d_{2}+U_{2}(t) d_{1}^{2} .
\end{aligned}
$$

From (46) and (48) it follows that

$$
\begin{aligned}
c_{1} & =-d_{1}, \\
2(1+\beta)^{2} 2^{2 k} a_{2}^{2} & =U_{1}^{2}(t)\left(c_{1}^{2}+d_{1}^{2}\right) .
\end{aligned}
$$

From (47), (49), and (51), we obtain

$$
a_{2}^{2}=\frac{U_{1}^{3}(t)\left(c_{2}+d_{2}\right)}{2\left[(1+2 \beta) 3^{k} U_{1}^{2}(t)-(1+\beta)^{2} 2^{2 k} U_{2}(t)\right]} .
$$

Using (10) and (25) for the coefficients $c_{2}$ and $d_{2}$, we immediately get the desired estimate on $\left|a_{2}\right|$ as asserted in (44).

By subtracting (49) from (47) and using (50) and (51), we get

$$
a_{3}=\frac{U_{1}^{2}(t)\left(c_{1}^{2}+d_{1}^{2}\right)}{2(1+\beta)^{2} 2^{2 k}}+\frac{U_{1}(t)\left(c_{2}-d_{2}\right)}{2(1+2 \beta) 3^{k}} .
$$

Again using (10) and (25) for the coefficients $c_{1}, c_{2}, d_{1}$, and $d_{2}$, we get the desired estimate on $\left|a_{3}\right|$ as asserted in (45). 
Remark 12. Let $f$ given by (1) be in the class $\mathscr{R}_{\Sigma}^{k}(\Phi(z, t))$. Then

$$
\begin{aligned}
& \left|a_{2}\right| \leq \frac{2 t \sqrt{2 t}}{\sqrt{\left|\left(3^{k}-2^{2 k}\right) 4 t^{2}+2^{2 k}\right|}}, \\
& \left|a_{3}\right| \leq \frac{4 t^{2}}{2^{2 k}}+\frac{2 t}{3^{k}} .
\end{aligned}
$$

Remark 13. Let $f$ given by (1) be in the class $\mathscr{H}_{\Sigma}^{k}(\Phi(z, t))$. Then

$$
\begin{aligned}
& \left|a_{2}\right| \leq \frac{2 t \sqrt{2 t}}{\sqrt{\left|\left(3^{k+1}-2^{2(k+1)}\right) 4 t^{2}+2^{2(k+1)}\right|}}, \\
& \left|a_{3}\right| \leq \frac{4 t^{2}}{2^{2(k+1)}}+\frac{2 t}{3^{k+1}} .
\end{aligned}
$$

By taking $k=0$ we deduce the following results.

Remark 14. Let $f$ given by (1) be in the class $\mathscr{F}_{\Sigma}(\beta, \Phi(z, t))$. Then

$$
\begin{aligned}
& \left|a_{2}\right| \leq \frac{2 t \sqrt{2 t}}{\sqrt{\left|(1+\beta)^{2}-4 t^{2} \beta^{2}\right|}} \\
& \left|a_{3}\right| \leq \frac{4 t^{2}}{(1+\beta)^{2}}+\frac{2 t}{(1+2 \beta)}
\end{aligned}
$$

$$
\begin{aligned}
& \left|a_{3}-\mu a_{2}^{2}\right| \\
& \leq\left\{\begin{array}{l}
\frac{t}{(1+2 \lambda) 3^{k}}, \\
\frac{8|1-\mu| t^{3}}{\left|\left(2(1+2 \lambda) 3^{k}-\left(\lambda^{2}+5 \lambda+2\right) 2^{2 k}\right) 4 t^{2}+(1+\lambda)^{2} 2^{2 k}\right|},
\end{array}\right.
\end{aligned}
$$$$
\begin{aligned}
& |\mu-1| \leq \frac{\left|(1+\lambda)^{2} 2^{2 k} / 4 t^{2}+2(1+2 \lambda) 3^{k}-\left(\lambda^{2}+5 \lambda+2\right) 2^{2 k}\right|}{2(1+2 \lambda) 3^{k}} \\
& |\mu-1| \geq \frac{\left|(1+\lambda)^{2} 2^{2 k} / 4 t^{2}+2(1+2 \lambda) 3^{k}-\left(\lambda^{2}+5 \lambda+2\right) 2^{2 k}\right|}{2(1+2 \lambda) 3^{k}}
\end{aligned}
$$

Remark 15. Let $f$ given by (1) be in the class $\mathscr{F}_{\Sigma}^{0}(1, \Phi(z, t)) \equiv$ $\mathscr{H}_{\Sigma}(\Phi(z, t))$. Then

$$
\begin{aligned}
& \left|a_{2}\right| \leq \frac{t \sqrt{2 t}}{\sqrt{\left|1-t^{2}\right|}} \\
& \left|a_{3}\right| \leq t^{2}+\frac{2 t}{3} .
\end{aligned}
$$

Remark 16. Let $f$ given by (1) be in the class $\mathscr{F}_{\Sigma}^{0}(0, \Phi(z, t)) \equiv$ $\mathscr{R}_{\Sigma}(\Phi(z, t))$. Then

$$
\begin{aligned}
& \left|a_{2}\right| \leq 2 t \sqrt{2 t}, \\
& \left|a_{3}\right| \leq 4 t^{2}+2 t .
\end{aligned}
$$

\section{Fekete-Szegö Inequality for the Function Classes $\mathscr{M}_{\Sigma}^{k}(\lambda, \Phi(z, t))$ and $\mathscr{F}_{\Sigma}^{k}(\lambda, \Phi(z, t))$}

Due to Zaprawa [18], in this section we obtain the FeketeSzegö inequality for the function classes $\mathscr{M}_{\Sigma}^{k}(\lambda, \Phi(z, t))$ and $\mathscr{F}_{\Sigma}^{k}(\beta, \Phi(z, t))$.

Theorem 17. Let $f$ given by (1) be in the class $\mathscr{M}_{\Sigma}^{k}(\lambda, \Phi(z, t))$ and $\mu \in \mathbb{R}$. Then one has
Proof. From (29) and (31)

$$
\begin{aligned}
a_{3} & -\mu a_{2}^{2}=(1-\mu) \\
& \cdot \frac{U_{1}^{3}(t)\left(c_{2}+d_{2}\right)}{\left(4(1+2 \lambda) 3^{k}-2(1+3 \lambda) 2^{2 k}\right) U_{1}^{2}(t)-2 U_{2}(t)(1+\lambda)^{2} 2^{2 k}} \\
& +\frac{U_{1}(t)\left(c_{2}-d_{2}\right)}{4(1+2 \lambda) 3^{k}}=U_{1}(t)\left[\left(h(\mu)+\frac{1}{4(1+2 \lambda) 3^{k}}\right) c_{2}\right. \\
& \left.+\left(h(\mu)-\frac{1}{4(1+2 \lambda) 3^{k}}\right) d_{2}\right],
\end{aligned}
$$

where

$$
\begin{aligned}
& h(\mu) \\
& =\frac{(1-\mu) U_{1}^{2}(t)}{2\left[\left(2(1+2 \lambda) 3^{k}-(1+3 \lambda) 2^{2 k}\right) U_{1}^{2}(t)-(1+\lambda)^{2} 2^{2 k} U_{2}(t)\right]} .
\end{aligned}
$$

Then, in view of (10), we conclude that

$$
\begin{aligned}
& \left|a_{3}-\mu a_{2}^{2}\right| \\
& \quad \leq \begin{cases}\frac{t}{(1+2 \lambda) 3^{k}}, & 0 \leq|h(\mu)| \leq \frac{1}{4(1+2 \lambda) 3^{k}} \\
4 t|h(\mu)|, & |h(\mu)| \geq \frac{1}{4(1+2 \lambda) 3^{k}} .\end{cases}
\end{aligned}
$$

Taking $\mu=1$, we have the following corollary.

Corollary 18. If $f \in \mathscr{M}_{\Sigma}^{k}(\lambda, \Phi(z, t))$, then

$$
\left|a_{3}-a_{2}^{2}\right| \leq \frac{t}{(1+2 \lambda) 3^{k}}
$$


Corollary 19. Let $f$ given by (1) be in the class $\mathcal{S}_{\Sigma}^{k}(\Phi(z, t))$ and $\mu \in \mathbb{R}$. Then one has

$$
\begin{array}{ll}
\left|a_{3}-\mu a_{2}^{2}\right| & \\
\leq \begin{cases}\frac{t}{3^{k}}, & |\mu-1| \leq \frac{\left|2^{2 k} / 8 t^{2}+3^{k}-2^{2 k}\right|}{3^{k}} \\
\frac{8|1-\mu| t^{3}}{\left|\left(3^{k}-2^{2 k}\right) 8 t^{2}+2^{2 k}\right|}, & |\mu-1| \geq \frac{\left|2^{2 k} / 8 t^{2}+3^{k}-2^{2 k}\right|}{3^{k}} .\end{cases}
\end{array}
$$

Corollary 20. Let $f$ given by (1) be in the class $\mathscr{K}_{\Sigma}^{k}(\Phi(z, t))$ and $\mu \in \mathbb{R}$. Then one has

$$
\left|a_{3}-\mu a_{2}^{2}\right| \leq \begin{cases}\frac{t}{3^{k+1}}, & |\mu-1| \leq \frac{\left|2^{2 k} / 2 t^{2}+3^{k+1}-2^{2(k+2)}\right|}{3^{k+1}} \\ \frac{2|1-\mu| t^{3}}{\left|\left(3^{k+1}-2^{2(k+2)}\right) 2 t^{2}+2^{2 k}\right|}, & |\mu-1| \geq \frac{\left|2^{2 k} / 2 t^{2}+3^{k+1}-2^{2(k+2)}\right|}{3^{k+1}} .\end{cases}
$$

Particularly, for $\mu=1$ if $f \in \mathscr{K}_{\Sigma}^{0}(\Phi(z, t))$ one obtains

$$
\left|a_{3}-a_{2}^{2}\right| \leq \frac{t}{3}
$$

$$
\begin{aligned}
\mid a_{3} & -\mu a_{2}^{2} \mid \\
& \leq\left\{\begin{array}{l}
\frac{2 t}{(1+2 \beta) 3^{k}}, \\
\frac{8|1-\mu| t^{3}}{\left|\left[(1+2 \beta) 3^{k}-(1+\beta)^{2} 2^{2 k}\right] 4 t^{2}+(1+\beta)^{2} 2^{2 k}\right|},
\end{array}\right.
\end{aligned}
$$$$
\begin{aligned}
& |\mu-1| \leq \frac{\left|(1+\beta)^{2} 2^{2 k} / 4 t^{2}+(1+2 \beta) 3^{k}-(1+\beta)^{2} 2^{2 k}\right|}{(1+2 \beta) 3^{k}} \\
& |\mu-1| \geq \frac{\left|(1+\beta)^{2} 2^{2 k} / 4 t^{2}+(1+2 \beta) 3^{k}-(1+\beta)^{2} 2^{2 k}\right|}{(1+2 \beta) 3^{k}}
\end{aligned}
$$

Theorem 21. Let $f$ given by (1) be in the class $\mathscr{F}_{\Sigma}^{k}(\beta, \Phi(z, t))$ and $\mu \in \mathbb{R}$. Then one has
Proof. From (29) and (31)

$$
\begin{aligned}
a_{3} & -\mu a_{2}^{2}=(1-\mu) \\
& \cdot \frac{U_{1}^{3}(t)\left(c_{2}+d_{2}\right)}{2\left[(1+2 \beta) 3^{k} U_{1}^{2}(t)-(1+\beta)^{2} 2^{2 k} U_{2}(t)\right]} \\
& +\frac{U_{1}(t)\left(c_{2}-d_{2}\right)}{2(1+2 \beta) 3^{k}}=U_{1}(t) \\
& \cdot\left[\left(h(\mu)+\frac{1}{2(1+2 \beta) 3^{k}}\right) c_{2}\right. \\
& \left.+\left(h(\mu)-\frac{1}{2(1+2 \beta) 3^{k}}\right) d_{2}\right],
\end{aligned}
$$

where

$$
h(\mu)=\frac{(1-\mu) U_{1}^{2}(t)}{2\left[(1+2 \beta) 3^{k} U_{1}^{2}(t)-(1+\beta)^{2} 2^{2 k} U_{2}(t)\right]} .
$$

Then, in view of (10), we conclude that

$$
\begin{aligned}
& \left|a_{3}-\mu a_{2}^{2}\right| \\
& \quad \leq \begin{cases}\frac{2 t}{(1+2 \beta) 3^{k}}, & 0 \leq|h(\mu)| \leq \frac{1}{2(1+2 \beta) 3^{k}} \\
4 t|h(\mu)|, & |h(\mu)| \geq \frac{1}{2(1+2 \beta) 3^{k}} .\end{cases}
\end{aligned}
$$

Taking $\mu=1$, we have the following corollary.

Corollary 22. If $f \in \mathscr{F}_{\Sigma}^{k}(\beta, \Phi(z, t))$, then

$$
\left|a_{3}-a_{2}^{2}\right| \leq \frac{2 t}{(1+2 \beta) 3^{k}}
$$

Corollary 23. Let $f$ given by (1) be in the class $\mathscr{R}_{\Sigma}^{k}(\Phi(z, t))$ and $\mu \in \mathbb{R}$. Then one has

$$
\begin{array}{ll}
\left|a_{3}-\mu a_{2}^{2}\right| & \\
\leq \begin{cases}\frac{2 t}{3^{k}}, & |\mu-1| \leq \frac{\left|2^{2 k} / 4 t^{2}+3^{k}-2^{2 k}\right|}{3^{k}} \\
\frac{8|1-\mu| t^{3}}{\left|\left[3^{k}-2^{2 k}\right] 4 t^{2}+2^{2 k}\right|}, & |\mu-1| \geq \frac{\left|2^{2 k} / 4 t^{2}+3^{k}-2^{2 k}\right|}{3^{k}} .\end{cases}
\end{array}
$$


Particularly, for $\mu=1$ if $f \in \mathscr{R}_{\Sigma}^{0}(\Phi(z, t))$ one obtains

$$
\left|a_{3}-a_{2}^{2}\right| \leq 2 t
$$

$$
\left|a_{3}-\mu a_{2}^{2}\right| \leq \begin{cases}\frac{2 t}{3^{k+1}}, & |\mu-1| \leq \frac{\left|2^{2 k} / t^{2}+3^{k+1}-2^{2(k+2)}\right|}{3^{k+1}} \\ \frac{2|1-\mu| t^{3}}{\left|\left[3^{k+1}-2^{2(k+2)}\right] t^{2}+2^{2 k}\right|}, & |\mu-1| \geq \frac{\left|2^{2 k} / t^{2}+3^{k+1}-2^{2(k+2)}\right|}{3^{k+1}} .\end{cases}
$$

Corollary 24. Let $f$ given by (1) be in the class $\mathscr{H}_{\Sigma}^{k}(\Phi(z, t))$ and $\mu \in \mathbb{R}$. Then one has

12] G. S. Salagean, "Subclasses of univalent functions," in Proceedings of the 5th Romanian Finnish Seminar in Complex Analysis, pp. 362-372, Bucharest, Romania, 1983.

[13] G. Murugusundaramoorthy, C. Selvaraj, and O. S. Babu, "Coefficient estimates for Pascu-type subclasses of bi-univalent functions based on subordination," International Journal of Nonlinear Science, vol. 19, no. 1, pp. 47-52, 2015.

[14] S. Altinkaya and S. Yalcin, "On the Chebyshev polynomial bounds for classes of univalent functions," Khayyam Journal of Mathematics, vol. 2, no. 1, pp. 1-5, 2016.

[15] J. Dziok, R. K. Raina, and J. Sokół, "Application of Chebyshev polynomials to classes of analytic functions," Comptes Rendus Mathematique, vol. 353, no. 5, pp. 433-438, 2015.

[1] P. L. Duren, "Univalent Functions," in Grundlehren der Mathematischen Wissenschaften, Band 259, Springer-Verlag, New York, NY, USA, 1983.

[2] E. H. Doha, "The first and second kind chebyshev coefficients of the moments for the general order derivative on an infinitely differentiable function," International Journal of Computer Mathematics, vol. 51, no. 1-2, pp. 21-35, 1994.

[3] J. C. Mason, "Chebyshev polynomial approximations for the L-membrane eigenvalue problem," SIAM Journal on Applied Mathematics, vol. 15, pp. 172-186, 1967.

[4] D. A. Brannan, J. Clunie, and W. E. Kirwan, "Coefficient estimates for a class of star-like functions," Canadian Journal of Mathematics, vol. 22, pp. 476-485, 1970.

[5] D. A. Brannan and T. S. Taha, "On some classes of bi-univalent functions," Studia Universitatis Babeş-Bolyai: Mathematica, vol. 31, no. 2, pp. 70-77, 1986.

[6] M. Lewin, "On a coefficient problem for bi-univalent functions," Proceedings of the American Mathematical Society, vol. 18, pp. 63-68, 1967.

[7] E. Netanyahu, "The minimal distance of the image boundary from the origin and the second coefficient of a univalent function in $\mathrm{z}<1$," Arch. Ration. Mech. Anal, vol. 32, pp. 100-112, 1969.

[8] H. M. Srivastava, A. K. Mishra, and P. Gochhayat, "Certain subclasses of analytic and bi-univalent functions," Applied Mathematics Letters, vol. 23, no. 10, pp. 1188-1192, 2010.

[9] Q.-H. Xu, H. M. Srivastava, and Z. Li, "A certain subclass of analytic and close-to-convex functions," Applied Mathematics Letters, vol. 24, no. 3, pp. 396-401, 2011.

[10] X.-F. Li and A.-P. Wang, "Two new subclasses of bi-univalent functions," International Mathematical Forum, vol. 7, no. 29-32, pp. 1495-1504, 2012.

[11] K. Vijaya, M. Kasthuri, and G. Murugusundaramoorthy, "Coefficient bounds for subclasses of bi-univalent functions defined by the Salagean derivative operator," Boletin de la Asociaciton Matematica Venezolana, 2014.
16] B. A. Frasin and M. K. Aouf, "New subclasses of bi-univalent functions," Applied Mathematics Letters, vol. 24, no. 9, pp. 15691573, 2011.

[17] T. Panigrahi and G. Murugusundaramoorthy, "Coefficient bounds for bi-univalent analytic functions associated with Hohlov operator," Proceedings of the Jangjeon Mathematical Society. Memoirs of the Jangjeon Mathematical Society, vol. 16, no. 1, pp. 91-100, 2013.

[18] P. Zaprawa, "On the Fekete-Szegö problem for classes of bi-univalent functions," Bulletin of the Belgian Mathematical Society - Simon Stevin, vol. 21, no. 1, pp. 169-178, 2014. 


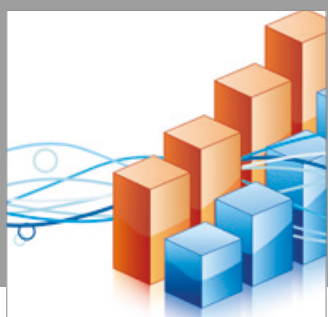

Advances in

Operations Research

vatersals

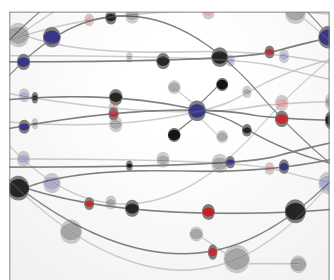

\section{The Scientific} World Journal
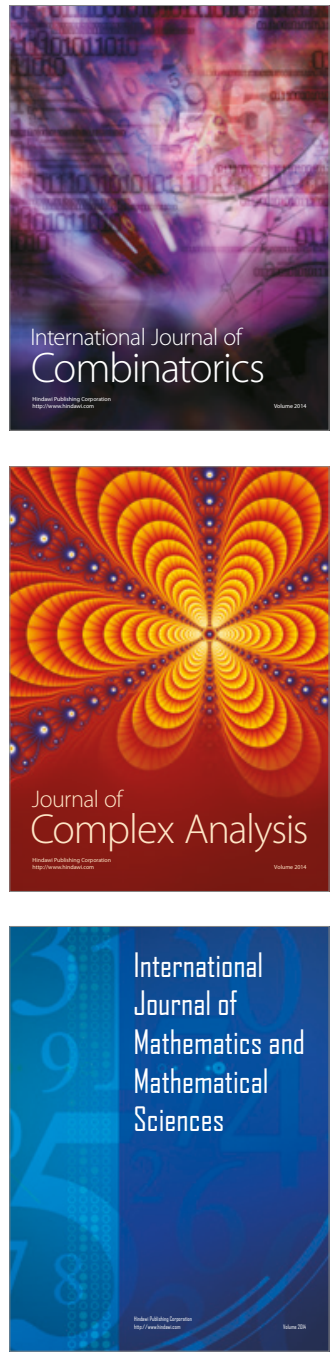
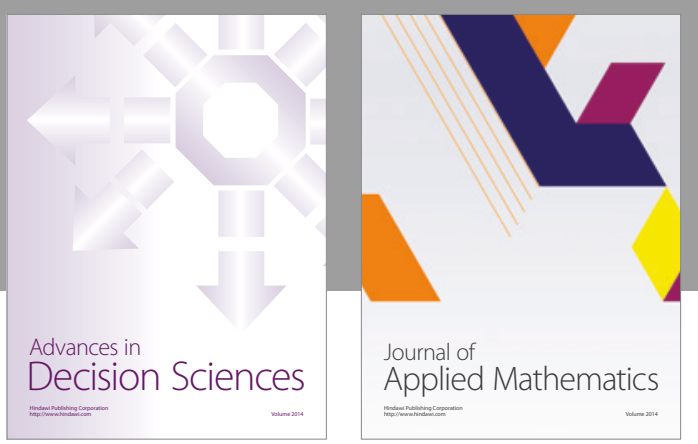

Algebra

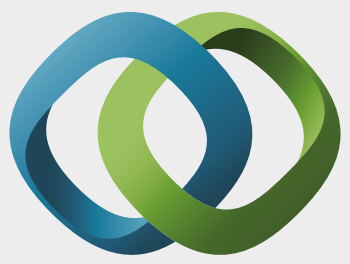

\section{Hindawi}

Submit your manuscripts at

https://www.hindawi.com
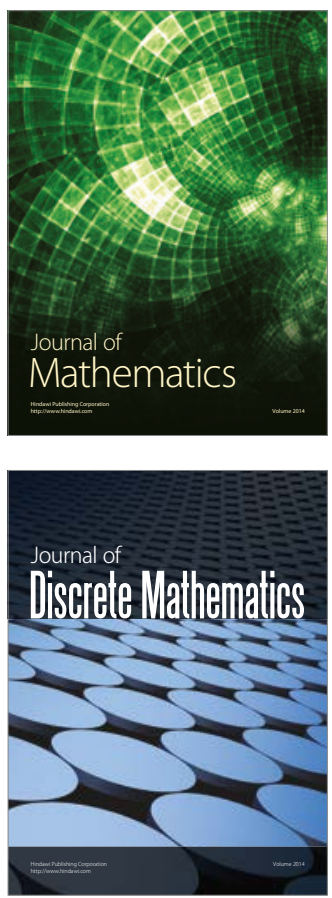

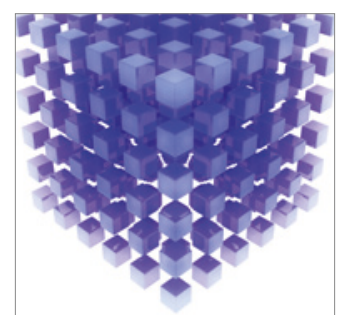

Mathematical Problems in Engineering
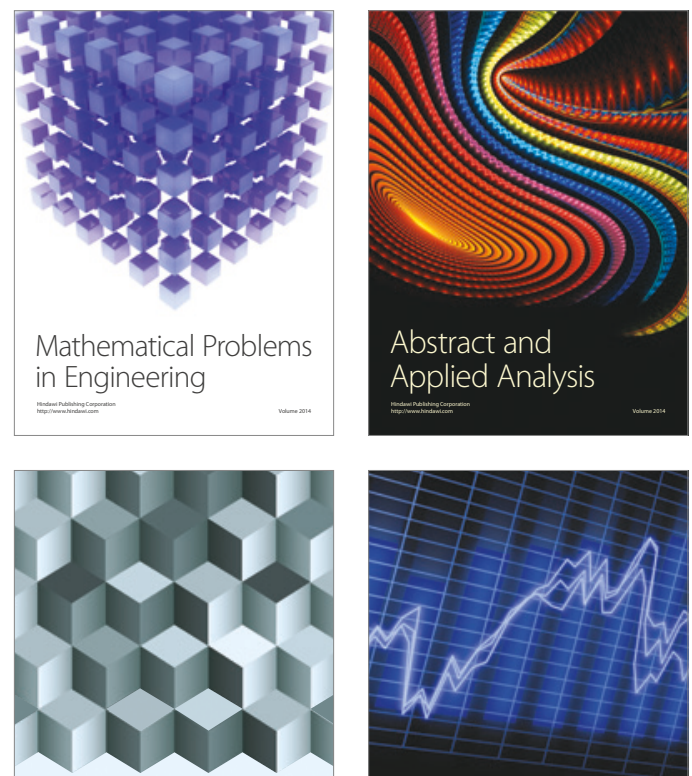

Journal of

Function Spaces

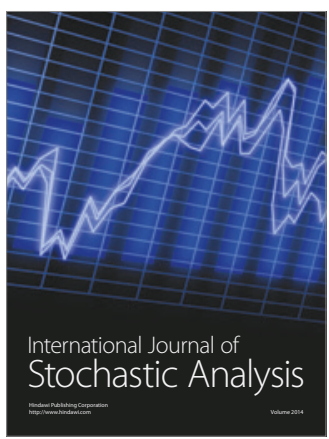

Probability and Statistics
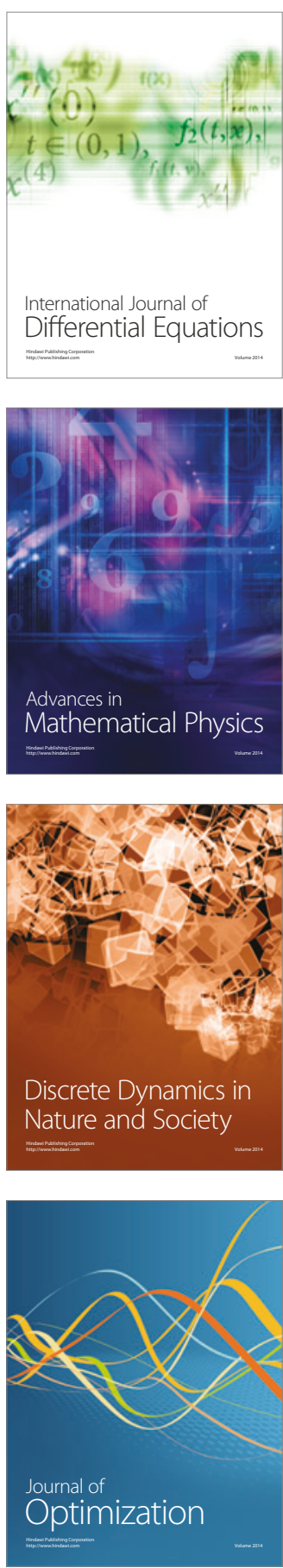